\title{
Effect of Vitamin D Supplementation in Improving Pregnancy Rates in Infertile Women with PCOS
}

M.A.Nosseir, A.M.Sadek, S.H.Awad and T.I.Edris

Obstetrics and Gynecology,Dept.,Faculty of Medicine,Benha Univ.,Benha, Egypt

E.Mail:shimaa.hamada20@outlook.com

\begin{abstract}
Polycystic ovarian condition (PCOS) is the most well-known endocrine problem that influences ladies in the youngster bearing period. The point of the current examination was to contemplate the impact of nutrient $\mathrm{D}$ supplementation in nutrient D inadequate PCOS ladies, as respects the clinical pregnancy and ovulation rates. Patient and techniques : This is a Randomized controlled clinical preliminary investigation included 100 nutrient D inadequate/lacking fruitless ladies with an analysis of PCOS. Serum 25-OH-VD levels were estimated and ladies were haphazardly partitioned into Vitamin D and placepo gatherings. Following 3 months of metformin \pm nutrient D supplementation, all included ladies got ovulation enlistment by letrozole $2.5 \mathrm{mg}$. Sequential trans vaginal ultrasound was performed and evaluated. Results: Following nutrient D3 supplementation, the rate of continuous pregnancy demonstrated genuinely huge expansion in nutrient D gathering contrasted with fake treatment group $(39.1 \%$ and $18.4 \% \%$ separately) $\mathrm{P}=0.025$. Likewise measurably critical expansion in ovulation after the enlistment cycles in vit D gathering contrasted with the fake treatment gathering ( $\mathrm{p}$ $=0.001,0.001$ and 0.045$)$ through three continuous acceptance cycles . End: In subfertile ladies with PCOS, Vitamin D supplementation may have a possible adjuvant part to standard enlistment of ovulation regimens.
\end{abstract}

Keywords: Vitamin D, Infertile Women, PCOS.

\section{Introduction}

Polycystic ovarian condition (PCOS) is the most well-known endocrine issue that influences ladies in the kid bearing period [1].

Anovulation is perhaps the most noticeable highlights of such a condition, and is a typical reason for female barrenness [2]

A few investigations and a new meta-examination have exhibited a high pervasiveness of nutrient $D$ insufficiency among ladies with PCOS, with almost 67$85 \%$ having serum 25-OH-nutrient D under $20 \mathrm{ng} / \mathrm{ml}$ [3]

Low degrees of serum 25-OH-nutrient $\mathrm{D}$ was demonstrated to be related with heftiness, expanded insulin obstruction, testosterone, and dehydroepiandrosterone sulfate (DHEAS) [4].

This NAFLD may clarify nutrient D insufficiency as the initial step of enactment of nutrient $\mathrm{D}$ happens inside the liver [5]

Nutrient D supplementation was appeared by a few examinations to improve metabolic and biochemical boundaries of ladies with PCOS and metabolic disorder, glucose, insulin, DHEAS, aggregate and free testosterone, androstenedione levels were decreased [6]. Lamentably, not many examinations have investigated the effect of nutrient $\mathrm{D}$ inadequacy just as nutrient $\mathrm{D}$ supplementation of the clinical results of PCOS; to be specific ovulation and pregnancy rates .

The point of the current examination was to consider the impact of nutrient D supplementation in nutrient D lacking PCOS ladies, as respects the clinical pregnancy and ovulation rates.

\section{Patient and Method}

This is a Randomized controlled clinical preliminary investigation included 100 nutrient D lacking/inadequate barren ladies with an analysis of PCOS. arbitrarily assigned into one of two gatherings .
Incorporation measures was $20-35$ years of age, Women with essential or optional barrenness (characterized as failure to imagine in spite of continuous conjugal life for at any rate a year). Conclusion of PCOS, as per presence of in any event two of the three Rotterdam's standards [oligo/amenorrhea, clinical or biochemical hyper androgenism and sonographic polycystic ovaries]. Nutrient $\mathrm{D}$ inadequacy or deficiency (characterized as serum 25-OH-nutrient D levels $<20$ $\mathrm{ng} / \mathrm{ml}$ or $20-<30 \mathrm{ng} / \mathrm{ml}$, individually).

\subsection{Exclusion criteria was}

Ladies with thyroid issues, hyper prolactinemia or different reasons for hyper androgenism (for example Cushing syndrome).Patients experiencing Chronic illnesses, for example, ongoing renal disappointment, cirrhosis, pancreatitis, nephrotic condition and threat, past ovarian medical procedure, Couples who have other related reasons for barrenness (for example male factor infertility(abnormal semen boundary dependent on WHO (2010) ) or long length of fruitlessness (> 5 years) that would require helped proliferation, Women with a weight file (BMI) $\square 35 \mathrm{Kg} / \mathrm{m} 2$, con $\neg$ genital or obtained uterine distortions, drugs consump 7 tion that would influence digestion and Vitamin D absorp $\neg$ tion, for example, Carbamazepine and Phenobarbital, Phenytoin and Body mass list higher $35 \mathrm{~kg} / \mathrm{m} 2$ and . The examination populace was a sequential arrangement of sub fruitful ladies (age somewhere in the range of 20 and 35 years), withPCOS (analyzed by utilizing ESHRE/ASRM standards) Rotterdam's rules 2.2All participants had at least two of the three criteria.

1. Ovulatory dysfunction (oligo-/anovulation), [defined as fewer than eight episodes of menstrual bleeding per year or menses that occur at intervals greater than 35 days [7]

2. Hyperandrogenism (Clinical hyper androgenism [defined by the presence of hirsutism (assessed by a modified Ferriman-Gallwey score $\geq 8$ [8], acne or 
androgenic alopecia (Rotterdam ESHRE/ASRMSponsored PCOS Consensus Workshop Group, 2003)]and/or biochemical signs of hyper androgenism[defined by elevated free androgen index(RCOG Green-top guideline no. 33, 2014)] (elevated testosterone level $\geq 2.5 \mathrm{nmol} / \mathrm{l}$ ), and/or

3. ultrasound finding of polycystic ovaries (defined by an increased number of small antral follicles $\geq 12$ follicles $(2-9 \mathrm{~mm})$ and/or an ovarian volume of $>10$ $\mathrm{cm} 3$ (obtained from the formula $\left[0.5_{\text {_ }}\right.$ length $~$ width thickness]) in one or both ovaries that was performed by the principal investigator to avoid inter observer variability.

Different issues that mirror PCOS, including thyroid infection and prolactin overabundance, grown-up beginning adrenal hyperplasia were precluded. All members had demonstrated in any event one fallopian tube patency and typical semen examination of their male accomplices .

We rejected sub fruitful ladies because of causes other than PCOS, ladies with history of a half year or less from past treatment with any of the sex steroids, fruitlessness drugs, insulin sensitizers, or some other hormonal treatment and ladies with excessive touchiness to any prescription was utilized in the investigation .

At first 120 couples were met dependent on Inclusion standards .20 couples were ex $\neg$ cluded dependent on rejection models. The entirety of the members entered the examination subsequent to giving composed educated assent and were permitted to leave the investigation at any ideal time. . Benchmark Vitamin $\mathrm{D}$ status was estimated by evaluating circling levels of $25 \mathrm{OH}-\mathrm{VD}$ in serum tests by Fasting blood tests were gathered by venipuncture prior to beginning the examination .

Serum 25-OH-VD levels were estimated utilizing the superior fluid chromatography (HPLC)- based Chrom frameworks demonstrative pack. (25-OH nutrient D level was estimated utilizing chemiluminescent immunoassay utilizing business units (LIAISON® 25OH Vitamin D TOTAL Assay, DiaSorin, MN)

All patients results were Vitamin D inadequacy or deficiency (characterized as serum 25-OH-nutrient D levels $<20 \mathrm{ng} / \mathrm{ml}$ or $20-<30 \mathrm{ng} / \mathrm{ml}$, individually) [9]

100 females were haphazardly isolated into Vitamin D and placepo bunches based PC created or irregular designation programming with one square . Members of Vitamin D gathering got a week by week portion of 50000 units of Vitamin D supplementation Breva (VordexPHARM). While the benchmark group got: a fake treatment tablet for 12 weeks as pearls orally.both bunches got metformin (Glucophage ${ }^{\circledR} 1000 \mathrm{mg}$ XR [Merck Serono, Darmstadt, Germany]) once each day with breakfast feast for a very long time prior to beginning enlistment of ovulation .

Boxes containing Vitamin D and Placebo rings were la $\neg$ beled dependent on irregular assignment number, aside from the two people distributing the Vitamin D and fake treatment, partici-pants, clinicians were all incognizant in regards to the examination. The no. of assigned gathering is to be covered in sequentially numbered obscure and fixed envelopes that solitary delivered after enlistment .

Organization of Vitamin D or fake treatment began the second day of the last feminine time frame (LMP.Or withdrawal seeping (following 5-day treatment with oral noresthisterone $5 \mathrm{mg}$ [Steronate ${ }^{\circledR}$, Hi-Pharm, Egypt] two times a day).

For Women of gathering I who got nutrient D supplementation notwithstanding metformin treatment Serum vit D re examine for bunch 1 was finished .

Following 3 months of metformin \pm nutrient D supplementation, all included ladies got ovulation acceptance by letrozole $2.5 \mathrm{mg}$ [Femara ${ }^{\circledR}$, Novartis, Egypt] two times every day, from day 2 through day 6 of menses or withdrawal seeping (following 5-day treatment with oral noresthisterone $5 \mathrm{mg}$ [Steronate $\AA$, Hi-Pharm, Egypt] two times a day)

Sequential trans vaginal ultrasound was performed utilizing VSON (E6) transvaginal test recurrence 4$9 \mathrm{MHz}$ to screen the number and size of creating follicles to season of human chorionic gonadotropin (hCG) administration(Choriomon ${ }^{\circledR}$ IBSA) 5000 IU ampoule, single dose10,000 IU intramuscular which was given when the main follicle arrives at $18-20 \mathrm{~mm}$ in measurement to instigate ovulation. All members were exhorted about coordinated intercourse during the treatment cycles .

The essential result was Positive progressing clinical pregnancy, characterized as presence of practical intrauterine pregnancy following 12 weeks of development .

Positive ovulation, which is considered to have happened if the follicle arrives at a mean breadth of 18 $25 \mathrm{~mm}$ and therefore changed in size, shape, or sonographic thickness. The adjustments in ultrasound picture of the follicle that cracks are: vanishing or abrupt decline in size, expanded echogenicity, abnormality of follicular divider, as well as appearance of free liquid in the pocket of Doglas.

No. of develop predominant follicle(s), which is characterized as a follicle with a mean breadth of in any event $18 \mathrm{~mm}$.

Positive biochemical pregnancy, characterized as certain serum pregnancy test after a missed period or 16 days after finding of ovulation.

Positive clinical pregnancy, characterized as presence of intrauterine practical gestational sac at 7 weeks of growth .

Treatment proceeded until pregnancy or for up to three acceptance cycles. Members who neglected to ovulate and stayed amenorrheic for $\geq 6$ weeks, withdrawal draining was instigated with norethisterone treatment.

\section{Results}

No statistically significant differences were found between the two groups regarding the age, type and duration of infertility. and base line serum vitamin D level. 
Table (1) Comparison between the studied groups according to age, BMI, duration of infertility and primary VitD level.

\begin{tabular}{lccccccc}
\hline & \multicolumn{2}{c}{ Case group (46) } & \multicolumn{2}{c}{ Placebo group (49) } & \multirow{2}{*}{ Statistical test (st t) } & P value \\
\cline { 2 - 5 } & Mean & SD & Mean & SD & & & 0.06 \\
Age & 25.76 & 4.7 & 27.57 & 4.66 & & 1.89 & 0.68 \\
BMI & 27.88 & 4.37 & 28.45 & 3.73 & & 1.14 & 0.50 \\
Duration of infertility & 2.5 & 1.30 & 2.21 & 1.15 & & 0.26 \\
Primary vitD & 10.51 & 3.41 & 11.71 & 3.54 & & 1.69 & 0.09 \\
\hline
\end{tabular}

30 patients of 46 investigated still have Insufficient or inadequate nutrient $\mathrm{D}$ levels in spite of supplementation however serum nutrient D levels in reassay estimations indicated critical distinction $\mathrm{P}$ esteem $<0.001^{* *}$.

16 patients of 46 dissected in nutrient $\mathrm{D}$ gathering acquired typical serum nutrient $\mathrm{D}$ levels with mean $(34.34 \mathrm{ng} / \mathrm{ml})$ which indicated critical contrast( $\mathrm{P}$ esteem $<0.001 * *$ )in contrast with gauge levels with mean $(11.46 \mathrm{ng} / \mathrm{ml})$.

Following 3 months of metformin \pm nutrient D supplementation, all remembered ladies for the two gatherings got ovulation acceptance by letrozole $2.5 \mathrm{mg}$ [Femara®, Novartis, Egypt] two times every day, from day 2 through day 6 of menses or withdrawal seeping (following 5-day treatment with oral noresthisterone 5 mg [Steronate ${ }^{\circledR}$, Hi-Pharm, Egypt] two times a day).

As to estimated results (enrolled after 3 patterns of ovulation acceptance or get pregnant ) results were as following

(18/46)PCOSwomen in the intercession group $(39.1 \%)$ looked at to(9/49)PCOS lady in the fake treatment gathering $(18.4 \%)$ had accomplished on going pregnancy, characterized as presence of practical intrauterine pregnancy following 12 weeks of gestation.p $(0.025)$ this distinction considered measurably critical. A P esteem $<0.05$

(25/46 ) PCOS ladies in mediation gathering $(54.3 \%)$ and (9/49) PCOS ladies in placepo group $(18.4 \%)$ Got fruitful Ovulation first monthP value $<0.001 * *$. $(29 / 39)$ in intercession gathering $(74.4 \%)$ and $(12 / 46)$ in placepo gathering $(26.1 \%)$ Got fruitful Ovulation second monthP value $<0.001 * *$. (22/34) in intercession gathering (64.7\%) and (19/45) in placepo gathering (42.2\%) Got effective Ovulation third monthP value $<0.001^{* *}$. As just ( 5/49)PCOS ladies neglected to ovulate for three acceptance cycles in the intercession gathering (10.9\%) versus( $21 / 46$ ) ladies in the placepo gathering ( $42.9 \%)$.

Positive serum pregnancy test was acquired in $(21 / 46)$ in intercession gathering $(45.7 \%)$ while it was acquired in(16/49) in placepo gathering (32.7\%) (P estimation of 0.19 ).

Ladies who had clinical pregnancy in intercession bunch were (18/46) (39.1\%) while those in placepo bunch were (11/49) $(22.4 \%)$ with P estimation of 0.078 . No of gestational sacs didn't contrast statically between the two gatherings.

(3/21 )ladies had unsuccessful labor in intercession group $(14.3 \%)$ versus ( $7 / 16)$ in placepo group $(43.7 \%)$ Patients in mediation bunch had been partitioned agreeing serum vit $\mathrm{D}$ re test into two sub gatherings.

Lacking or inadequate nutrient $\mathrm{D}$ level notwithstanding treatment (30/46) (sub gathering A) and ordinary nutrient D level after supplementation (16/46)( subgroup B).There was no measurement contrast between the two gatherings in age, BMI, essential nutrient D level or type and length of barrenness, yet they varied statically in nutrient $\mathrm{D}$ re examine results were mean $(21.17 \mathrm{ng} / \mathrm{ml})$ and $(34.34 \mathrm{ng} / \mathrm{ml})$ in sub gathering An and subgroup B individually $\mathrm{p}=<0.001 *$.

As to essential result( on going pregnancy) in subgroup $\mathrm{B}$ ( 11/16) patients accomplished on going pregnancy versus (7/30) in sub gathering A which varied altogether $\mathrm{p}$ esteem $0.003 * *$

The second out comes likewise contrasted fundamentally between two gatherings:

Ovulation in first month was(12/30) in subgroup sometime 13/16 in subgroup B with $\mathrm{P}$ esteem 0.007 . Ovulation in second month was (17/27) in subgroup sometime $12 / 12$ in subgroup B with a $\mathrm{P}$ esteem 0.017

Ovulation in third month was (13/25) in subgroup sometime $9 / 9$ in subgroup $B$ with a $\mathrm{P}$ esteem 0.013 . No of prevailing follicles, Positive biochemical pregnancy and Positive clinical pregnancy all contrasted statically between two gatherings with $\mathrm{P}$ esteem $0.027,0.022$ and 0.003 individually

There was no statistic difference between two groups according No of gestational sacs and miscarriage rate.

Table (2) Comparison between cases subgroups according to results of ovulation induction.

\begin{tabular}{|c|c|c|c|c|c|c|}
\hline & \multicolumn{2}{|c|}{$\begin{array}{l}\text { Insufficient deficient after } \\
\text { ttt (30) SUBGROUP(A) }\end{array}$} & \multicolumn{2}{|c|}{$\begin{array}{l}\text { Normal after ttt (16) } \\
\text { SUBGROUP (B) }\end{array}$} & \multirow{2}{*}{$\begin{array}{l}\text { Statistical } \\
\text { test }\left(x^{2}\right)\end{array}$} & \multirow[b]{2}{*}{$P$ value } \\
\hline & No & $\%$ & No & $\%$ & & \\
\hline \multicolumn{7}{|c|}{ Type of infertility } \\
\hline 1ry & 17 & 56.7 & 10 & 62.5 & & \\
\hline 2 ry & 13 & 43.3 & 6 & 37.5 & 0.15 & 0.70 \\
\hline
\end{tabular}




\begin{tabular}{|c|c|c|c|c|c|c|}
\hline \multicolumn{7}{|c|}{ Ongoing pregnancy } \\
\hline Positive & 7 & 23.3 & 11 & 68.8 & & \\
\hline Negative & 23 & 76.7 & 5 & 31.3 & 9.04 & $0.003 * *$ \\
\hline \multicolumn{7}{|c|}{ Ovulation $1^{\text {st }}$ month } \\
\hline Positive & 12 & 40.0 & 13 & 81.2 & & \\
\hline Negative & 18 & 60.0 & 3 & 18.8 & 7.16 & $0.007 * *$ \\
\hline \multicolumn{7}{|c|}{ Ovulation $2^{\text {nd }}$ month } \\
\hline Positive & $17 / 27$ & 63.0 & $12 / 12$ & 100 & & \\
\hline Negative & $10 / 27$ & 37.0 & $0 / 12$ & 0.0 & $\mathrm{FET}=4.19$ & $0.017^{*}$ \\
\hline \multicolumn{7}{|c|}{ Ovulation $3^{\text {rd }}$ month } \\
\hline Positive & $13 / 25$ & 52.0 & 9/9 & 100 & & \\
\hline Negative & $12 / 25$ & 48.0 & $0 / 9$ & 0.0 & $\mathrm{FET}=4.74$ & $0.013 *$ \\
\hline \multicolumn{7}{|c|}{ No of dominant follicles } \\
\hline $\mathbf{0}$ & 5 & 16.7 & 0 & 0.0 & & \\
\hline 1 & 19 & 63.3 & 7 & 43.8 & & \\
\hline 2 & 3 & 10.0 & 7 & 43.8 & & \\
\hline 3 & 3 & 10.0 & 2 & 12.5 & $\mathrm{FET}=8.26$ & $0.027 *$ \\
\hline \multicolumn{7}{|c|}{ Positive serum preg test } \\
\hline+ & 10 & 33.3 & 11 & 68.8 & & \\
\hline . & 20 & 66.7 & 5 & 31.3 & 5.28 & $0.022 *$ \\
\hline \multicolumn{7}{|c|}{ Positive clinical pregnancy } \\
\hline+ & 7 & 23.3 & 11 & 68.8 & & \\
\hline 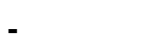 & 23 & 76.7 & 5 & 31.3 & 9.04 & $0.003 * *$ \\
\hline \multicolumn{7}{|c|}{ No of gestational sacs (18) } \\
\hline 1 & 6 & 85.7 & 9 & 81.8 & & \\
\hline 2 & 1 & 14.3 & 2 & 18.2 & $\mathrm{FET}=0.0$ & 1.0 \\
\hline
\end{tabular}

\section{Discussion}

To the most awesome aspect our insight, the current examination is one of only a handful few twofold visually impaired RCT to evaluate the impact of VD supplementation on clinical pregnancy rates and ovulation rate in PCOS patients going through ovarian enlistment by letrozole. In this examination, impact of nutrient D supplementation was evaluated on achievement of letrozole acceptance of ovulation and clinical pregnancy. 100 ladies determined to have polycystic ovary condition with nutrient $\mathrm{D}$ inadequacy or in adequacy were randomized into either two gatherings: Vitamin D gathering, including 50 ladies and placepo bunch including 50 ladies followed by acceptance of ovulation utilizing letrozole after nutrient D supplementation .

In this investigation, following nutrient D3 supplementation, the occurrence of progressing pregnancy demonstrated measurably critical expansion in nutrient $\mathrm{D}$ gathering contrasted with fake treatment group $(39.1 \%$ and $18.4 \% \%$ individually) $\mathrm{P}=0.025$.

As indicated by Firouzabadi et al., [10] For a situation control study, 100 fruitless PCOS ladies dependent on a haphazardly separated into two gatherings. Gathering I $(\mathrm{n}=50)$ were treated with metformin $1500 \mathrm{mg} / \mathrm{day}$, and gathering II $(\mathrm{n}=50)$ treated with metformin $1500 \mathrm{mg} /$ day in addition to Calcium $1000 \mathrm{mg} / \mathrm{day}$ and Vitamin D $100000 \mathrm{IU} / \mathrm{month}$ for a half year. They found that BMI diminished essentially ( $25.49 \pm 1.88$ versus $26.28 \pm 2.15$, p: 0.054 ) in gathering II. A superior improvement was acquired in managing feminine variations from the norm $(70 \%$ versus $58 \%$, p: 0.211$)$, follicle development ( $28 \%$ versus
$22 \%$, p: 0.698$)$, and barrenness (18\% versus $12 \%$, p: 0.401 ) in gathering II contrasted and gathering I, however these outcomes were not measurably critical. Nutrient D inadequacy was rewarded in $74 \%$ of the PCOS patients who had taken calcium and nutrient D supplementation. There was no relationship among's BMI and 25-OH-VD when the treatment ( $\mathrm{p} \geq 0.01)$.

In our investigation, $\mathrm{VD}$ bunch indicated an increment in both the clinical and biochemical pregnancy rates contrasted with fake treatment $39.1 \%$ and $45.7 \%$ versus $22.4 \%$ and 32.7 separately), albeit this didn't arrive at measurable importance $(\mathrm{p}=0.078$ and 0.19 individually).

In accordance with our stydy [11] in their investigation at Ain Shams University during the time frame between January 2018 and September 2018. discovered that clinical and bio substance pregnancy rates expanded in nutrient $\mathrm{D}$ gathering over the placepo gathering yet was not measurably huge $(\mathrm{p}=0.141$ and 0.105 separately) .

We have found likewise that $54.3 \%, 74.4 \%$ and $64.7 \%$ of ladies got VD had effective ovulation after the enlistment cycles contrasted and $18.4 \%, 26.1 \%$ and 42.2 $\%$ in the fake treatment gathering $(\mathrm{p}=0.001,0.001$ and 0.045 ) through three sequential acceptance cycles which is genuinely critical increment.

In accordance with this study [11] have discovered that $92.5 \%$ of ladies got VD had effective ovulation after the enlistment cycles contrasted and $78.5 \%$ in the fake treatment gathering $(p=0.007)$. The mean distance across of the prevailing follicle was $18.07 \pm 1.37 \mathrm{~mm}$ in the treatment gathering and was $16.79 \pm 3.00 \mathrm{~mm}$ in placepo bunch with $\mathrm{p}<0.001$. 
This discoveries were predictable with the aftereffects of [12] in their optional examination of the Pregnancy in PCOS I (PPCOS I) multicenter RCT found that the ovulation fluctuated straightforwardly with VD levels in inspected associate $(68 \%, 77 \%$, and $78 \%$ in those with VD lack, deficiency, and typical status, separately; $\mathrm{p}=0.050)$. With huge better ovulation rate with VD levels $\geq 20 \mathrm{ng} / \mathrm{mL}(\mathrm{p}=0.006)$. Also, VD level was fundamentally higher in ladies accomplished live birth (LB) contrasted with those weak with achieve LB with every $1 \mathrm{ng} / \mathrm{mL}$ increment in serum VD improved the probability of LB by $2 \%$ (OR, $1.02 ; 95 \%$ CI, 1.00 , $1.04 ; \mathrm{p}=0.046)$

Wehr et al., [13] found that VD treatment for 12 weeks in ladies with PCOS improved feminine abnormality in $30.4 \%$ of ladies and 4 out of 16 ladies looking for pregnancy at standard imagined (25\%).

Ott et al., [14] found that VD lack was related with lower paces of follicle advancement and pregnancy afterCC incitement.

Additionally Shahrokhi et al., [15] proposed apossible part of VD supplementation in subfertile PCOSwomen who go through ovarian incitement.

In our study,No. of develop prevailing follicle(s) which is characterized as a follicle with a mean measurement of in any event $18 \mathrm{~mm}$ varied essentially between gatherings. As just ( 5/49)PCOS ladies neglected to ovulate for three acceptance cycles in the intercession group $(10.9 \%)$ versus( $21 / 46$ ) ladies in the placepo gathering ( $42.9 \%$ ).

Likewise Rashidi et al., [16] found that the quantity of predominant follicles during the patterns of treatment was higher in the calcium-nutrient $\mathrm{D}$ in addition to metformin bunch than in either treatment alone $(p=0.03)$

In this pilot study, 60 fruitless PCOS patients were selected a randomized clinical preliminary and separated into three equivalent gatherings. Gathering 1 got 1,000 $\mathrm{mg}$ of calcium and 400 IU of nutrient D each day, orally. Gathering 2 got equivalent to Group 1, in addition to $1,500 \mathrm{mg} /$ day of metformin. Gathering 3 got 1,500 $\mathrm{mg} /$ day of metformin. The patients were treated for a very long time and followed up for a further 3 months. Routineness of menses, number of huge follicles $(\geq 14$ $\mathrm{mm}$ ) and pregnancy rates were looked at among the three gatherings. Summed up assessing condition tests demonstrated that the quantity of prevailing follicles $(\geq$ $14 \mathrm{~mm}$ ) during the 2-3 months of follow-up was higher in the calcium-nutrient $\mathrm{D}$ in addition to metformin bunch than in both of the other two gatherings $(p=0.03)$. they reasoned that the impacts of metformin and calciumnutrient $\mathrm{D}$ in controlling the period propose that they could likewise be viable for the treatment of anovulation and oligomenorrhea, with potential ramifications for pregnancy rates in PCOS patients.

Abedi et al., [17] in their clinical preliminary investigation led at Isfahan University of Medical Sciences and did at fruitless couples that alluded to Isfahan Fertility and Infertility Center from March 2016 to June 2016 and candi $\neg$ date of ICSI. Female with age going from 18 to 38 years who had Vitamin D level under $30 \mathrm{ng} / \mathrm{ml}$ without indication of Vitamin D insufficiency partook in the examination.

Consequences of the investigation of [17] showed that devouring Viᄀtamin D supplementation could be powerful in improving the clinical result of ICSI. In view of writing this ef $\neg$ fect is probably going to be ascribed to nearby impact of Vita-min D on endometrium .The clashing outcomes about progress in total ovulation Rate following nutrient $\mathrm{D}$ supplementation may mirror the complex heterogenous atomic imperfections fundamental hormonal and ovulatory disturbance in PCOS ladies.

It appears to be that the anovulatory imperfection in aportion of PCOS ladies is identified with interruption in nutrient Dsignaling; while the leftover bit have other fundamental anovulatory sub-atomic deformity. To additionally entangle the image, not all the upset nutrient Dsignaling-related PCOS parcel is relied upon to profit by nutrient $\mathrm{D}$ supplementation, perhaps because of the revealed impacts of VDR polymorphisms on the different hormonal and metabolic boundaries in PCOS ladies [13].

Till symptomatic devices for the atomic peril in PCOS ladies are accessible, we will depend on clinical forecast: disturbed nutrient D flagging related PCOS are bound to be experienced in nutrient D lacking PCOS ladies; while ladies with determined anovulatory imperfection notwithstanding typical nutrient D status are thought to generally have an anovulatory sub-atomic deformity separated from distorted nutrient D motioning In the 2016 auxiliary examination of the PPCOS I (Pregnancy in Polycystic Ovary Syndrome) multicenter randomized controlled preliminary, [12] and his associates evaluated serum 25OHD levels in the put away examples of the 626 members in the PPCOS I preliminary and connected them with the ovulatory reaction to enlistment of ovulation with clomiphene citrate alone or in mix with metformin. Nutrient D inadequate ladies were essentially less inclined to accomplish OV contrasted with those with 25OHD levels $\geq 20 \mathrm{ng} / \mathrm{ml}(\mathrm{P}=0.006)$.

The likelihood of accomplishing ovulation changed straightforwardly with nutrient D status $(68 \%, 77 \%, 78 \%$ in those with nutrient $\mathrm{D}$ inadequacy, deficiency and typical status, $\mathrm{P}=0.05$ ) [12].

As per the investigation of [12] serum 25OHD was altogether higher in ladies accomplishing live birth contrasted with those neglecting to achieve live birth $(25.34 \pm 10.39$ versus $23.16 \pm 9.71$ separately, $\mathrm{P}=$ 0.046). Every $1 \mathrm{ng} / \mathrm{ml}$ increment in 25OHD improved the probability of live birth by $2 \%$. Notwithstanding, this affiliation was evident at serum 250HD levels that are well past the edge of $30 \mathrm{ng} / \mathrm{ml}$ that is at present considered as an objective "ordinary" level. Most elevated probability for live birth was apparent in ladies with serum $25 \mathrm{OHD}$ level $>45 \mathrm{ng} / \mathrm{ml}$; interestingly, $25 \mathrm{OHD}$ levels $<20 \mathrm{ng} / \mathrm{ml}(<50 \mathrm{nmol} / \mathrm{L})$, were prescient of a hosed ovulatory reaction to ovulation enlistment systems. In view of these perceptions, [12] recommended that circling $25 \mathrm{OHD}$ degree of $45 \mathrm{ng} / \mathrm{ml}$ or higher ought 
to be considered as "ideal" for ladies endeavoring to imagine; accordingly, presenting the ideas of unmistakable "conceptive limits" of nutrient D underneath which ovulatory reaction to acceptance of ovulation is blunted (Lower Reproductive ThresholdLRT $<20 \mathrm{ng} / \mathrm{ml}$ ) and past which (Upper ReproductiveThreshold-URT $>45 \mathrm{ng} / \mathrm{ml}$ ) probability of live birth might be advanced and penchant for pregnancy misfortune is diminished. Outstandingly, the noticed URT is higher than 25OHD degrees of $20 \mathrm{ng} / \mathrm{ml}$ and 30 $\mathrm{ng} / \mathrm{ml}$ that are recognized by the Institute of Medicine and the Endocrine Society [9] separately, to reflect ordinary nutrient D status. Be that as it may, a significant impediment of the above finishes of [12] is the little quantities of members in their examination with 25OHD levels over the predefined edges for the characterized results, ie, just 10/540 (2\%) had 25OHD level >45 ng/ml (related with LB); consequently, the chance of alpha blunders being reflected in the noticed affiliations is conceivable.

\section{Conclusion}

Another significant angle portrayed in this investigation is that nutrient $\mathrm{D}$ supplementation in nutrient D insufficient PCOS ladies was noted to improve total ovulation rate.

These information.

\section{References}

[1] W.A.March, V.M.Moore, K.J.Willson, D.I.Phillips, R.J.Norman, M.J.Davies. The prevalence of polycystic ovary syndrome in a community sample assessed under contrasting diagnostic criteria. Hum Reprod.vol. 25,pp.54451,2010 .

[2] I.F.Stein, M.R.Cohen. Surgical treatment of bilateral polycystic ovaries. Am J Obstet Gynecol.vol.38,pp.465, 1939.

[3] S.Liangpunsakul, N,Chalasani. Serum vitamin D concentrations and unexplained elevation in ALT among US adults. Dig Dis Sci.vol.56,pp.21242129,2011.

[4] P.T.RahimLiu, S.Stenger, H.Li, L.Wenzel, B.H.Tan, S.R.Krutzik. Tolllike receptor triggering of a vitamin $\mathrm{D}$ mediated human antimicrobial response. Science.vol.311,pp.1770-1773,2006.

[5] G.Ponchon, A.L.Kennan, H.F.DeLuca. Activation of vitamin $\mathrm{D}$ by the liver. J Clin Invest.vol.48,pp.2032-2037,1969.

[6] E.Bargiota Diamanti-Kandarakis. The effects of old, new and emerging medicines on metabolic aberrations in PCOS,"Therapeutic Advances in Endocrinology andMetabolism,vol. 3,pp. 2747,2012 .
[7] R.J.Norman, D.Dewailly, R.S.Legro, T.E.Hickey. Polycystic ovary syndrome. Lancet.vol. 370,pp.685-97,2007.

[8] R.Yildizhan, M.Kurdoglu, E.Adali, A.Kolusari, B.Yildizhan, HG.Sahin , M.Kamaci: Serum 25hydroxyvitamin D concentrations in obese and nonobese women with polycystic ovary syndrome. GynecolObstet.vol.280,PP.559-63,2009.

[9] M.F.Holick, N.C.Binkley, H.A.Bischoff-Ferrari. Evaluation, treatment, and prevention of vitaminD deficiency: an Endocrine Society clinical practice guideline. J Clin Endocrinol Metab .vol.96,pp.1911-1930,2011.

[10]R.d.Firouzabadi, A.Aflatoonian, S.Modarresi, L.Sekhavat, S.MohammadTaheri. Therapeutic effects of calcium \& vitamin D supplementation in women with PCOS. Complement Ther Clin Pract. May.vol.18(2),pp.85-8,2012.

[11] Rasheedy, Radwa \& Sammour, Hazem \& Elkholy, Abdellatif \& Salim, Yasmine. The efficacy of vitamin D combined with clomiphene citrate in ovulation induction in overweight women with polycystic ovary syndrome: a double blind, randomized clinical trial. Endocrine.vol. 69,pp.112-130, 2020

[12]Pal, H. Zhang, J.Williams. Vitamin D status relates to reproductive outcome in women with polycystic ovary syndrome: secondary analysis of a multicenter randomized controlled trial. J. Clin. Endocrinol. Metab.vol. 101(8),pp. 3027-3035 (2016).

[13] Wehr, T.R.Pieber, B.Obermayer-Pietsch, Effect of vitamin D3 treatment on glucose metabolism and menstrual frequency in polycystic ovary syndrome women: a pilot study. J. Endocrinol.Investig .vol.34(10),pp.757-763,2011.

[14] Ott, L.Wattar, C.Kurz, R.Seemann, J.C.Huber, K.Mayerhofer, E.Vytiska-Binstorfer, Parameters for calcium metabolism in women with polycystic ovary syndrome who undergo clomiphene, 2013.

[15] Shahrokhi, F.Ghaffari, F. Kazerouni, Role of vitamin D in female reproduction. Clin. Chim. Acta .vol.45,pp.33-38,2016.

[16] Rashidi, F.Haghollahi, M.Shariat, F. Zayerii, The effects of calcium-vitamin D and metformin on polycystic ovary syndrome: a pilot study. Taiwan J. Obstet. Gynecol.vol.48(2),pp.142-147 ,2009.

[17] S.Abedi, M.Taebi, M.H.Nasr Esfahani. Effect of vitamin D supplementation on intracytoplasmic sperm injection outcomes: a randomized doubleblind placebo-controlled trial. Int $\mathrm{J}$ Fertil Steril.vol.13(1),pp.18-23. D,2019. 